\title{
Admissibility Estimation of Burr Type XI Distribution Under Entropy Loss Function Based on Record Values
}

\section{Lanping Li}

Department of Basic Subjects, Hunan University of Finance and Economics, Changsha, China

\section{Email address:}

lilanping1981@163.com

\section{To cite this article:}

Lanping Li. Admissibility Estimation of Burr Type XI Distribution Under Entropy Loss Function Based on Record Values. American Journal of Theoretical and Applied Statistics. Vol. 5, No. 6, 2016, pp. 348-353. doi: 10.11648/j.ajtas.20160506.13

Received: September 21, 2016; Accepted: October 1, 2016; Published: October 25, 2016

\begin{abstract}
The aim of this paper is to study the estimation of parameter of Burr Type XI distribution on the basis of lower record values. First, the minimum variance unbiased estimator and maximum likelihood estimator are obtained. Then the Bayes and empirical Bayes estimators of the unknown parameter are derived under entropy loss function. Finally, the admissibility and inadmissibility of a class of inverse linear estimators are discussed.
\end{abstract}

Keywords: Admissibility, Bayes and Empirical Bayes Estimators, Record Values, Entropy Loss Function

\section{Introduction}

Burr distribution contains twelve forms, which first introduced by Burr in 1942 [1]. However, Burr type XII distribution is the most of the authors have considered [2-6], But other types of Burr distribution do not get much attention. Feroze and Aslam [7] studied the Bayes estimation of the parameter of Burr type XI distribution under the assumption of eight priors (informative, non-informative, single and mixture of priors). The entropy and precautionary loss functions were used in their Bayesian analysis. Feroze et al. [8] studied the Bayes estimation and prediction of the Burr type XI distribution based on censored samples on the basis of Five informative and non-informative priors under five different (symmetric and asymmetric) loss functions for posterior analysis.

This paper is devoted to study the Bayes estimation problem of the unknown parameter of the Burr type XI distribution with cumulative distribution function and probability density function given as follows, respectively (Feroze and Aslam [7]):

$$
\begin{array}{r}
F(x ; \theta)=\left\{x-\left(\frac{1}{2 \pi}\right) \sin (2 \pi x)\right\}^{\theta}, x>0 \\
f(x ; \theta) \propto \theta\left\{x-\left(\frac{1}{2 \pi}\right) \sin (2 \pi x)\right\}^{\theta}, x>0
\end{array}
$$

Here $\theta>0$ is the unknown parameter, $\propto$ denotes the probability density function omits the function of a proportionality constant with respect to the parameter $\theta$.

Record values is an important order statistics, which was first studied by Chandler in 1952 [9]. It has many application in various fields, such as weather, sports, economics, lifetests, stock market and so on, and the statistical inference study of record values has received great attention [10-13]. For example, Wang et al. [14] investigated the point estimation and confidence intervals estimation for the parameter of Weibull distribution based on record values. Arabi Belaghi et al. [15] considered several different types confidence intervals for the scale parameter of Burr type XII distribution based on upper record values. They put forward improved confidence intervals estimation based on the preliminary test technique, Thompson shrinkage technique and Bayesian approach. Barranco-Chamorro et al. [16] proposed two new maximum likelihood methods for estimating the unknown sample size in a simple random sample from an absolutely continuous population one the basis of record values.

The admissibility of estimators is an important topic in statistical inference. Many authors studied the admissibility and inadmissibility of various estimators for different distributions. For example, Wen and Levy [17] considered properties of Bayes estimators in a normal mean problem derived under a bounded loss function called BLINEX Loss function. Zakerzadeh and Zahraie [18] considered the 
dmissibility of estyimators in non-regular family under squared-log error loss function. Cao and kong [19] studied the general admissibility for a class of linear estimators in a general multivariate linear model under balanced loss function. More relevant reference can be found in [20-22].

The purpose of this paper is to study the estimation and admissible characters of estimators of the parameter of Burr type XI distribution.

\section{Preliminary Knowledge}

This section will first recall some definitions of record values. Then derive the maximum likelihood estimator (MLE) and minimum variance unbiased estimator of the parameter of Burr Type XI distribution.

Definition 1. Let $X_{1}, X_{2}, \cdots$ be a sequence of independent and identically distributed random variables. Suppose

$$
L(1)=1, L(n+1)=\min \left\{j: j>L(n), X_{j}<X_{L(n)}\right\}
$$

We say $X_{L(n)}, n=1,2, \cdots$ is a lower record value of this sequence.

Suppose we observe $\mathrm{n}$ lower record values $X_{L(1)}=x_{1}, X_{L(2)}=x_{2}, \cdots, X_{L(n)}=x_{n}$ from Burr Type $\mathrm{XI}$ distribution (1). Let $t$ is the observation of $T=-\ln \left[X_{L(n)}-\frac{1}{2 \pi} \sin \left(2 \pi X_{L(n)}\right)\right], \underline{x}=\left(x_{1}, x_{2}, \cdots, x_{n}\right)$ is the observation of $\underline{X}=\left(X_{L(1)}, X_{L(2)}, \cdots, X_{L(n)}\right)$. Then the likelihood function of $\underline{X}$ is given as follows (Arnold et al. [23])

$$
l(\theta)=f\left(x_{n} ; \theta\right) \prod_{i=1}^{n-1} \frac{f\left(x_{i} ; \theta\right)}{F\left(x_{i} ; \theta\right)} \propto \theta e^{-t \theta} \cdot \prod_{i=1}^{n-1} \theta=\theta^{n} e^{-t \theta}
$$

Then $X_{L(n)}$ is a sufficient statistics for the first $\mathrm{n}$ lower record values. The MLE can be obtained by solving the following log-likelihood equation

$$
\frac{d \ln l(\theta)}{d \theta}=0
$$

Then the MLE can be obtained as

$$
\hat{\theta}_{M L E}=\frac{n}{T}
$$

The marginal probability density function of $X_{L(n)}$ is given by

$$
\begin{gathered}
f_{n}\left(x_{n} ; \theta\right)=f\left(x_{n} ; \theta\right) \frac{R^{n-1}\left(x_{n} ; \theta\right)}{(n-1) !} \\
=\theta e^{-\theta t} \frac{(\theta t)^{n-1}}{(n-1) !}=\frac{\theta^{n}}{(n-1) !} t^{n-1} e^{-\theta t}
\end{gathered}
$$

Here $R(x, \theta)=-\ln (F(x, \theta))$.

According to the above equation, the probability density function of $T$ is

$$
f_{T}(t)=\frac{\theta^{n}}{\Gamma(n)} t^{n-1} e^{-\theta t}
$$

Further more, we have

$$
E\left(\hat{\theta}_{M L E}\right)=E\left(\frac{n}{T}\right)=\frac{n}{n-1} \theta
$$

then

$$
E\left[\frac{n-1}{T}\right]=E\left[\frac{n-1}{n} \hat{\theta}_{M L E}\right]=\theta
$$

thus $\frac{n-1}{T}$ is an unbiased estimator of $\theta$, and it is also a function the complete and sufficient statistics $T$. Therefore, by using Lehmann and Scheffe's theorem, the estimator $\frac{n-1}{T}$ is the minimum variance unbiased estimator of $\theta$, note it by

$$
\hat{\theta}_{U M V U}=\frac{n-1}{T}
$$

\section{Bayes Estimation}

Loss function is an important aspect in Bayesian analysis. This section will derive the Bayes estimatior under a useful asymmetric loss function named entropy loss function (Dey et al. [24]):

$$
L(\hat{\theta}, \theta)=\frac{\hat{\theta}}{\theta}-\ln \frac{\hat{\theta}}{\theta}-1
$$

The Bayes estimator under the entropy loss is denoted by $\hat{\theta}_{B E}$, given by

$$
\hat{\theta}_{B}=\left[E\left(\theta^{-1} \mid X\right)\right]^{-1}
$$

Suppose the prior distribution of parameter $\theta$ is Gamma distributions, $\Gamma(\alpha, \beta)$, with the following probability density function

$$
\pi(\theta \mid \alpha, \beta)=\frac{\beta^{\alpha}}{\Gamma(\alpha)} \theta^{\alpha-1} e^{-\beta \theta}, \theta>0
$$

where $\alpha>0$ and $\beta>0$ are the hyper parameters. Note that the limiting case $\alpha, \beta \rightarrow 0$ of the prior Gamma distribution equals to non-informative prior distribution $\pi(\theta) \propto \theta^{-1}$. 
According to equations (3) and (10), using Bayesian theorem, we can get the posterior probability density function as follows:

$$
\begin{aligned}
h(\theta \mid x) & \propto l(\theta) \cdot \pi(\theta ; \alpha, \beta) \\
& \propto \theta^{n} e^{-\theta t} \theta^{\alpha-1} e^{-\beta \theta} \\
& \propto \theta^{n+\alpha-1} e^{-(\beta+t) \theta}
\end{aligned}
$$

Then the posterior distribution of $\theta$ is $\Gamma(n+\alpha, T+\beta)$, and by equation (9), the Bayes estimator of $\theta$ under entropy loss function is

$$
\hat{\theta}_{B}=\left[E\left(\theta^{-1} \mid X\right)\right]^{-1}=\left(\frac{T+\beta}{n+\alpha-1}\right)^{-1}
$$

Thus

$$
\hat{\theta}_{B}=\left(\frac{1}{n+\alpha-1} T+\frac{\beta}{n+\alpha-1}\right)^{-1},
$$

which is of the form $(c T+d)^{-1}$

Remark 1. For the non-informative prior $\pi(\theta) \propto \theta^{-1}$, the posterior distribution of parameter $\theta$ is $\Gamma(n, T)$ and we obtain the generalized Bayes estimator

$$
\hat{\theta}_{*}=\frac{T}{n-1}
$$

Remark 2. Assume that the prior distributions $\Gamma(\alpha, \beta)$ has the unknown prior parameter $\beta$. Then we can use the empirical Bayes approach to estimate it. From equations (3) and (10), we calculate the marginal probability density function of $\underline{X}=\left(X_{L(1)}, \cdots, X_{L(n)}\right)$ as

$$
\begin{aligned}
& m(\underline{x} \mid \beta)=\int_{0}^{\infty} f(\underline{x} ; \theta) \pi(\theta \mid \beta) d \theta \\
& \propto \int_{0}^{\infty} \theta^{n} e^{-\theta t} \frac{\beta^{\alpha}}{\Gamma(\alpha)} \theta^{\alpha-1} e^{-\beta \theta} d \theta \\
& \propto \frac{\beta^{\alpha}}{\Gamma(\alpha)} \frac{\Gamma(n+\alpha)}{(\beta+t)^{n+\alpha}}
\end{aligned}
$$

Based on $m(\underline{x} \mid \beta)$, we can obtain an MLE estimator $\hat{\beta}$ of $\beta$ as $\hat{\beta}=\frac{\alpha}{n} T$

Then we obtain the empirical Bayes estimator by substituting $\hat{\beta}$ for in the Bayes estimator, That is

$$
\begin{aligned}
\hat{\theta}_{E B} & =\left(\frac{1}{n+\alpha-1} T+\frac{1}{n+\alpha-1} \frac{\alpha}{n} T\right)^{-1} \\
& =\left[\frac{n+\alpha}{n(n+\alpha-1)} T\right]^{-1}
\end{aligned}
$$

Obviously, the empirical Bayes estimator $\hat{\theta}_{E B}$ also have the inverse linear form $(c T+d)^{-1}$.

\section{Admissibility of $(c T+d)^{-1}$}

Note that the estimators obtained in Section 2 and Section 3 are all the special cases of the class of inverse linear estimators with the form $(c T+d)^{-1}$. This section supposes that:

(i) $X_{L(1)}=x_{1}, X_{L(2)}=x_{2}, \cdots, X_{L(n)}=x_{n}$ are $\mathrm{n}$ lower record values from Burr Type XI distribution with the distribution function (1).

(ii) Let $t$ is the observation of $T=-\ln \left[X_{L(n)}-\frac{1}{2 \pi} \sin \left(2 \pi X_{L(n)}\right)\right], \underline{x}=\left(x_{1}, x_{2}, \cdots, x_{n}\right)$

is the observation of $\underline{X}=\left(X_{L(1)}, X_{L(2)}, \cdots, X_{L(n)}\right)$.

(iii) We always let $c^{*}=\frac{1}{n-1}$.

In the rest of this section, all obtained estimators will be compared on the basis of their risks under the entropy loss function. We will also study the conditions under which general inverse linear estimators are admissible in terms of risk function.

Theorem 1. The estimator $(c T+d)^{-1}$ is admissible, provided $0 \leq c<c^{*}$ and $d>0$.

Proof. From equation (12), we know that the coefficient of $T$ is strictly between 0 and $c^{*}$ for every $\alpha>0$ and $\beta>0$, and the constant $\frac{\beta}{n+\alpha-1}$ is strictly larger than 0 . Then the inverse linear form $(c T+d)^{-1}$ is admissible for $0<c<c^{*}$ and $d>0$.

For the case $c=0, d>0$, the estimator $(c T+d)^{-1}$ is admissible since it is the unique Bayes estimator for which $R(\theta, d)=0$ when $\theta=d$.

Theorem 2. The inverse linear estimator $(c T+d)^{-1}$ is admissible, provided $c=c^{*}$ and $d \geq 0$.

Proof. We first consider the case $c=c^{*}$ and $d>0$. Let the parameter $\theta$ has the prior distribution $\pi_{k}(\theta)$ with the following density function

$$
\pi_{k}(\theta)=\frac{\beta^{1 / k}}{\Gamma(1 / \mathrm{k})} \theta^{1 / k-1} e^{-\beta \theta}, \beta>0, k>0
$$


Suppose that $A$ is a non-degenerate convex subset of $(0,+\infty)$, then it can be shown that there exists a constant $k_{0}$ satisfying $\int_{A} \pi_{k}(\lambda) d \lambda \geq \varepsilon$ for some $\varepsilon>0$ and all $k \geq k_{0}$.

In fact,

$$
\begin{aligned}
I & =\int_{A} \beta^{\frac{1}{k}} \cdot \theta^{\frac{1}{k}-1} \cdot e^{-\beta \theta} d \theta \\
& =\int_{A \cap(0,1)} \beta^{\frac{1}{k}} \cdot \theta^{\frac{1}{k}-1} \cdot e^{-\beta \theta} d \theta+\int_{A \cap(1,+\infty)} \beta^{\frac{1}{k}} \cdot \theta^{\frac{1}{k}-1} \cdot e^{-\beta \theta} d \theta
\end{aligned}
$$

Because

$$
\beta^{\frac{1}{k}} \rightarrow 1 \quad(k \rightarrow \infty)
$$

So for $k>k_{0}$, the former equation satisfies the following inequality

$$
I \geq \frac{1}{2}\left[\int_{A \cap(0,1)} \theta^{\frac{1}{k}-1} \cdot e^{-\beta \theta} d \theta+\int_{A \cap[1,+\infty)} \theta^{\frac{1}{k}-1} \cdot e^{-\beta \theta} d \theta\right]
$$

Let

$$
\varepsilon_{0}=\frac{1}{2}\left[\int_{A \cap(0,1)} \theta^{\frac{1}{k}-1} \cdot e^{-\beta \theta} d \theta+\int_{A \bigcap[1,+\infty)} \theta^{\frac{1}{k}-1} \cdot e^{-\beta \theta} d \theta\right]
$$

then the conclusion is proved.

The Bayes estimator with respect to $\pi_{k}(\theta)$ under the entropy loss function can be derived as in (11) which is given as

$$
\hat{\theta}_{k}=\left(\frac{T+\beta}{n+1 / k-1}\right)^{-1}
$$

Let $\hat{\theta}(X)=\left(\frac{T+\beta}{n-1}\right)^{-1}, F(\theta, t)$ is the joint probability density function of random vector $(\theta, T)$. Then we have

$$
d F(\theta, t)=\frac{\beta^{\frac{1}{k}}}{\Gamma(1 / \mathrm{k})} \theta^{1 / k-1} e^{-\beta \theta} \cdot \frac{\theta^{n}}{\Gamma(n)} t^{n-1} e^{-\theta t} d \theta d t
$$

The difference of the Bayes risks with respect to $\hat{\theta}_{k}$ and $\hat{\theta}$ can be derived as follows:

$$
\begin{aligned}
& r\left(\pi_{k}, \hat{\theta}\right)-r\left(\pi_{k}, \hat{\theta}_{k}\right)=E L(\hat{\theta}, \theta)-E L\left(\hat{\theta}_{k}, \theta\right) \\
& =\int_{0}^{+\infty} \int_{0}^{+\infty}\left(\frac{\hat{\theta}}{\theta}-\ln \frac{\hat{\theta}}{\theta}-1\right)-\left(\frac{\hat{\theta}_{k}}{\theta}-\ln \frac{\hat{\theta}_{k}}{\theta}-1\right) d F(\theta, t) \\
& =p(k) \int_{0}^{+\infty} \int_{0}^{+\infty} \frac{1}{\theta(T+\beta)} d F(\theta, t)-q(k)
\end{aligned}
$$

Where $\quad p(k)=\frac{-1}{k} \quad, \quad q(k)=\ln \frac{n-1}{n+1 / k-1} \quad$ and $p(k) \rightarrow 0, q(k) \rightarrow 0, k \rightarrow+\infty$
For any prior distribution $\Gamma(a, b)$ of $\theta$, the following conclusion is always tenable

$$
\begin{aligned}
& E\left[\frac{1}{\theta(T+\beta)}\right] \leq E\left[\frac{1}{\theta T}\right] \\
& =\int_{0}^{+\infty} \int_{0}^{+\infty} \frac{1}{\theta t} \cdot \frac{t^{n-1}}{\Gamma(n)} \theta^{n} e^{-\theta t} \cdot \frac{\beta^{\alpha}}{\Gamma(\alpha)} \theta^{\alpha-1} e^{-\beta \theta} d \theta d t \\
& =\int_{0}^{+\infty}\left[\int_{0}^{+\infty} t^{(n-1)-1} e^{-\theta t} d t\right] \cdot \frac{\beta^{\alpha}}{\Gamma(\alpha) \Gamma(\mathrm{n})} \theta^{(n+\alpha-1)-1} e^{-\beta \theta} d \theta \\
& =\int_{0}^{+\infty} \frac{\Gamma(n-1)}{\theta^{n-1}} \cdot \frac{\beta^{\alpha}}{\Gamma(\alpha) \Gamma(\mathrm{n})} \theta^{(n+\alpha-1)-1} e^{-\beta \theta} d \theta \\
& =\frac{\Gamma(n-1) \cdot \beta^{\alpha}}{\Gamma(\alpha) \Gamma(\mathrm{n})} \cdot \frac{\Gamma(\alpha)}{\beta^{\alpha}} \\
& =\frac{1}{n-1}<+\infty
\end{aligned}
$$

Thus

$$
\lim _{k \rightarrow \infty}\left(r\left(\pi_{k}, \hat{\theta}\right)-r\left(\pi_{k}, \hat{\theta}_{k}\right)\right)=0,
$$

Therefore, $(c T+d)^{-1}$ is admissible for any $\beta>0$ by Blyth's lemma. This proves that $(c T+d)^{-1}$ is also admissible for the case $c=c^{*}$ and $d>0$.

Now we prove the case $c=c^{*}$ and $d=0$. Obviously that the estimator $\left(c^{*} T\right)^{-1}$ is the limit of the Bayes estimator $\hat{\theta}_{B}=\left(\frac{1}{n+\alpha-1} T+\frac{\beta}{n+\alpha-1}\right)^{-1}$ relative to the gamma prior $\Gamma(\alpha, \beta)$, as $\alpha, \beta \rightarrow 0$. To prove the admissibility of the estimator $\left(c^{*} T\right)^{-1}$, it is easy to verify that the difference of Bayesian risk $r\left(\pi_{k},\left(c^{*} T\right)^{-1}\right)-r\left(\pi_{k}, \hat{\theta}_{B}\right)$ converges to zero as $\alpha, \beta \rightarrow 0$, which guarantees the admissibility of $\left(c^{*} T\right)^{-1}$.

Theorem 3 Let $(0,+\infty)$ be the parameter space and $[0,+\infty)$ be the action space. The inverse linear estimator $(c T+d)^{-1}$ is inadmissible under the entropy loss function (8) whenever one of the following conditions holds:

(i) $c<0$ or $d<0$;

(ii) $0<c \neq c^{*}, d=0$

(iii) $c>c^{*}$ and $d \geq 0$

Proof. For the case (i), we know that $(c T+d)^{-1}$ takes on negative values with positive probability. Therefore the inverse linear estimator $(c T+d)^{-1}$ is dominated by the estimator $\max \left(0,(c T+d)^{-1}\right)$ in this case. Then $(c T+d)^{-1}$ is inadmissible under case (i).

For the case (ii), the risk function of the estimator $(c T)^{-1}$ is 


$$
\begin{aligned}
R\left(\theta,(c T)^{-1}\right) & =E\left[\frac{1}{\theta(c T)}-\ln \frac{1}{\theta(c T)}-1\right] \\
& =\frac{1}{c \theta} E \frac{1}{T}+E(\ln T)+\ln \theta+\ln c-1
\end{aligned}
$$

According to equation (6), we can easily get the conclusions:

$$
\begin{gathered}
E \frac{1}{T}=\frac{\theta}{n-1}, \\
E(\ln T)=\psi(n)-\ln \theta
\end{gathered}
$$

Then derivative of the risk with respect to $c$ is

$$
\frac{\partial}{\partial c} R\left(\theta,(c T)^{-1}\right)=-\frac{1}{c^{2}} \frac{1}{n-1}+\frac{1}{c}=\frac{1}{c}\left(1-\frac{1 / c}{n-1}\right)
$$

Then

$$
\frac{\partial}{\partial c} R\left(\theta,(c T)^{-1}\right)<0 \text { where } 0<c<c^{*}=\frac{1}{n-1},
$$

and

$$
\frac{\partial}{\partial c} R\left(\theta,(c T)^{-1}\right)>0, \text { where } c>c^{*}
$$

Thus the risk of the estimator $(c T)^{-1}$ is minimized at $c=c^{*}$. Then the estimator $(c T)^{-1}$ is dominated by the estimator $\left(c^{*} T\right)^{-1}$, and thus $(c T+d)^{-1}$ is inadmissible under case (ii).

For the case (iii). The difference of risk functions between the inverse linear estimator $(c T+d)^{-1}$ and $\left(c^{*} T+\frac{c^{*}}{c} d\right)^{-1}$ are as follows:

$$
\begin{aligned}
& R\left(\theta,(c T+d)^{-1}\right)-R\left(\theta^{*},\left(c^{*} T+\frac{c^{*}}{c} d\right)^{-1}\right) \\
& =E\left[\frac{1}{\theta(c T+d)}-\ln \frac{1}{\theta(c T+d)}-\frac{1}{\theta\left(c^{*} T+\frac{c^{*}}{c} d\right)}+\frac{1}{\theta\left(c^{*} T+\frac{c^{*}}{c} d\right)}\right]
\end{aligned}
$$

In the condition (iii), $1-\frac{c}{c^{*}}<0$, then

$$
\begin{aligned}
& R(\theta, c T+d)-R\left(\theta^{*}, c^{*} T+\frac{c^{*}}{c} d\right) \\
& =\left\{E\left[\frac{1}{\theta(c T+d)}\left(1-\frac{c}{c^{*}}\right)+\ln \frac{c}{c^{*}}\right]\right\}, \\
& \left.\geq\left\{\left(1-\frac{c}{c^{*}}\right) E\left[\frac{1}{\theta c T}\right]+\ln \frac{c}{c^{*}}\right]\right\} \\
& =n\left(\frac{c^{*}}{c}-\ln \frac{c^{*}}{c}-1\right)>0
\end{aligned}
$$

Therefore, $R\left(\theta,(c T+d)^{-1}\right)$ is minimized at $c=c^{*}$. Then $(c T+d)^{-1}$ is dominated by $\left(c^{*} T+d\right)^{-1}$ in this case. Thus $(c T+d)^{-1}$ is inadmissible under case (iii).

Remark 3. By Theorem 3, the MLE estimators $\hat{\theta}_{M L E}$ and the empirical Bayes estimator $\hat{\theta}_{E B}$ are both inadmissible. They are dominated by the generalized Bayes estimator $\hat{\theta}_{*}=\left(c^{*} T\right)^{-1}$.

\section{Conclusion}

Admissibility of estimators is an important topic in statistical inference field. This paper considers the estimation of the unknown parameter from the Burr Type XI distribution on the basis of record values. The maximum likelihood estimator (MLE), minimum variance unbiased estimator, Bayes and empirical Bayes estimators are obtained. These estimators all belong to a general class of inverse linear estimators with the form $(c T+d)^{-1}$. The admissibility and inadmissibility of $(c T+d)^{-1}$ are studied under different conditions. As a result, the minimum variance unbiased estimator is admissible, but the empirical Bayes estimator and the maximum likelihood estimator are inadmissible

\section{Acknowledgement}

This study is partially supported by Natural Science Foundation of Hunan Province (No. 2015JJ3030) and Foundation of Hunan Educational Committee (No.15C0228).

\section{References}

[1] Burr W. I., 1942. Cumulative frequency distribution, Annals of Mathematical Statistics, 13 (217): 215-232.

[2] Abdel-Hamid A. H., 2009. Constant-partially accelerated life tests for Burr type-XII distribution with progressive type-II censoring, Computational Statistics \& Data Analysis, 53 (7): 2511-2523.

[3] Panahi H., Sayyareh A., 2014. Parameter estimation and prediction of order statistics for the Burr Type XII distribution with Type II censoring, Journal of Applied Statistics, 41 (1): 215-232.

[4] Tsai T. R., Lio Y., Jiang N., Fan Y. Y., 2015. Economical sampling plans with warranty based on truncated data from Burr type XII distribution, Journal of the Operational Research Society, 66 (9): 1511-1518.

[5] Wu J. W., Yu H. Y., 2005. Statistical inference about the shape parameter of the Burr type XII distribution under the failurecensored sampling plan, International Journal of Information \& Management Sciences, 163 (1): 443-482.

[6] Belaghi R. A., Arashi M., Tabatabaey S. M. M., 2014. Improved confidence intervals for the scale parameter of Burr XII model based on record values, Computational Statistics, 29 (5): 1153-1173. 
[7] Feroze, N., Aslam, M., 2012. Bayesian analysis of burr type xi distribution under single and mixture of priors, 2 (11): 487502 .

[8] Feroze N., Aslam M., Saleem A., 2014. Bayesian estimation and prediction of Burr type XI distribution under singly and doubly censored samples, International Journal of Hybrid Information Technology, 7 (2): 331-346.

[9] Chandler K. N., 1952. The distribution and frequency of record values, Journal of the Royal Statistical Society B, 14 (2): 220-228.

[10] Amin E. A., 2012. Bayesian and non-Bayesian estimation from type I generalized logistic distribution based on lower record values, Journal of Applied Sciences Research, 2012 (1): $118-126$.

[11] Selim M. A., 2012. Bayesian estimations from the twoparameter bathtub-shaped lifetime distribution based on record values, Pakistan Journal of Statistics \& Operation Research, 8 (2): 155-165.

[12] Zakerzadeh H., Jafari A. A., 2015, Inference on the parameters of two Weibull distributions based on record values, Statistical Methods \& Applications, 24 (1): 25-40.

[13] El-Sayed M. A., Abd-Elmougod G. A., Abdel-Khalek S., AbdElmougod G. A., Abdel-Khalek S., 2013. Bayesian and nonBayesian estimation of topp-leone distribution based lower record values, 45 (2): 133-145.

[14] Wang B. X., Ye Z. S., Wang B. X., Ye Z. S., 2015. Inference on the Weibull distribution based on record values. Computational Statistics \& Data Analysis, 83: 26-36.

[15] Arabi Belaghi, R., Arashi M., Tabatabaey S., 2014. Improved confidence intervals for the scale parameter of Burr XII model based on record values. Computational Statistics, 29 (5): 1153-1173.
[16] Barranco-Chamorro I., Moreno-Rebollo J. L., JiménezGamero M. D., Alba-Fernández M. V., 2015. Estimation of the sample size based on record values. Mathematics \& Computers in Simulation, 55 (118): 58-72.

[17] Wen D. L., Levy M. S., 2006. Admissibility of bayes estimates under BLINEX loss for the normal mean problem. Communications in Statistics-Theory and Methods, 30 (1): 155-163.

[18] Zakerzadeh H., Zahraie S. H. M., 2015. Admissibility in nonregular family under squared-log error loss. Metrika, 78 (2): 227-236.

[19] Cao, M. X., \& Kong, F. C. (2013). General admissibility for linear estimators in a general multivariate linear model under balanced loss function. Acta Mathematica Sinica, 29 (29): 1823-1832.

[20] Hara, H., Takemura, A., 2009. Bayes admissible estimation of the means in poisson decomposable graphical models. Journal of Statistical Planning \& Inference, 139 (4): 1297-1319.

[21] Kim B. H., Meeden G., 2007. Admissible estimation in an one parameter nonregular family of absolutely continuous distributions. Statistical Papers, 48 (2): 337-345.

[22] Mahmoudi E., 2012. Admissible and minimax estimators of $\theta$ with truncated parameter space under squared-log error loss function. Communication in Statistics-Theory and Methods, 41 (7): 1242-1253.

[23] Arnold, B. C., Balakrishnan, N., Nagaraja, H. N., 1998. Records. New York: John Wiley \& Sons.

[24] Dey D. K., Ghosh M. and Srinivasan C., 1987. Simultaneous estimation of parameters under entropy loss, J. Statist. Plan. and Infer., 15: 347-363. 